University of Nebraska - Lincoln

DigitalCommons@University of Nebraska - Lincoln

May 2000

\title{
The influence of both coordination number and lattice constant on the nonmetal to metal transition
}

I.N. Yakovkin

National Academy of Sciences of Ukraine, Prospect Nauki 46, Kiev 252022, Ukraine

Peter A. Dowben

University of Nebraska-Lincoln, pdowben@unl.edu

Follow this and additional works at: https://digitalcommons.unl.edu/physicsdowben

Part of the Physics Commons

Yakovkin, I.N. and Dowben, Peter A., "The influence of both coordination number and lattice constant on the nonmetal to metal transition" (2000). Peter Dowben Publications. 164.

https://digitalcommons.unl.edu/physicsdowben/164

This Article is brought to you for free and open access by the Research Papers in Physics and Astronomy at DigitalCommons@University of Nebraska - Lincoln. It has been accepted for inclusion in Peter Dowben Publications by an authorized administrator of DigitalCommons@University of Nebraska - Lincoln. 


\title{
The influence of both coordination number and lattice constant on the nonmetal to metal transition
}

\author{
I. N. Yakovkin \\ Institute of Physics, National Academy of Sciences of Ukraine, Prospect Nauki 46, Kiev 252022, Ukraine \\ P. A. Dowben \\ Department of Physics and Astronomy and the Center for Materials Research and Analysis, \\ Behlen Laboratory of Physics, University of Nebraska-Lincoln, Lincoln, Nebraska 68588-0111
}

(Received 29 April 1999; accepted 11 February 2000)

\begin{abstract}
We show that both coordination and lattice constant can have an important influence on the nonmetal to metal transition and the two parameters are not easily separated. Using example theoretical calculations for barium, we provide a compelling case that atomic coordination is a critical factor in determining the critical lattice constant for the nonmetal to metal transition. A comparison between the nonmetal to metal transition three-dimensional and two-dimensional systems is not possible on the basis of the atomic coordination alone. This is discussed in the context of a comparison of the available experimental data for both elemental expanded fluids (three-dimensional) and overlayers (quasi-two-dimensional). (c) 2000 American Institute of Physics. [S0021-9606(00)71217-7]
\end{abstract}

\section{INTRODUCTION}

It has been suggested that coordination number is critical for the nonmetal to metal transition in both alkali metals ${ }^{1,2}$ and mercury. ${ }^{3-5}$ It has been noted that for some systems, like Cs (Ref. 1) and Hg, 6,7 the critical atomic coordination numbers for the nonmetal to metal transition transcend dimensionality, seemingly applicable to both overlayers and expanded three dimensional fluids and even free clusters.

Neutron diffraction data is now available for expanded liquid $\mathrm{Hg}$ (Ref. 8) and Cs. ${ }^{9}$ There is some uncertainty as to the average coordination number $n$ for the onset of metallic behavior due to the difficulty in correctly interpreting the pair correlation function. Nonetheless, the onset of metallicity (at a critical density for $\mathrm{Hg}$ of about $\rho=9$ to $11 \mathrm{~g} \mathrm{~cm}^{-3}$ ) occurs at an average coordination number of about 5-6 (Ref. 8) for $\mathrm{Hg}$. This compares well with the onset of metallicity occurring at a critical coordination number of $n>5$ for $\mathrm{Hg}$ on $\mathrm{Cu}(100),{ }^{3} n \geqslant 7$ for $\mathrm{Hg}$ on $\mathrm{Ni}(111),{ }^{10}$ and $n \approx 6$ for $\mathrm{Hg}$ on $\mathrm{W}(110){ }^{11,12}$ While fully metallic behavior in $\mathrm{Hg}$ clusters occurs free clusters of 70 (Refs. 13,14 ) to 110 atoms in size, ${ }^{15}$ corresponding to a coordination number of $9-20,{ }^{16}$ the deviation from nonmetallic behavior and the transition toward metallic behavior begins at a coordination number of about 6. $^{11-14}$

For Cs, the critical coordination number in the bulk expanded fluid is seen to be about $3,{ }^{9}$ which has also been proposed as the critical coordination number for Cs overlayers on GaAs. ${ }^{1}$ As noted by Freeman and March, ${ }^{1}$ the agreement in critical coordination number for these very different Cs systems suggests that a localized chemical bonding picture may be, in part, applicable on the nonmetal side of the nonmetal to metal transition.

Ultimately what is at issue is the question whether the nonmetal to metal transition in ultrathin film overlayers and expanded liquid metals can be compared? If coordination number does have a profound influence on determining the critical behavior of the nonmetal to metal transition then there must be a strong influence of the "pair' potential on the critical point for the nonmetal to metal transition. ${ }^{1,4}$ As noted for mercury, ${ }^{5-7,10,17,18}$ coordination number is not the only criterion for metallicity across the nonmetal to metal transition; lattice constant is also a key factor.

Because the coordination in expanded liquid metals is reduced well below the values expected for a closed packed three-dimensional lattice, theory is necessary to test the importance of coordination on the critical lattice constant for the nonmetal to metal transition. This experimental complication makes it difficult to probe the electronic structure for a nonmetal to metal transition system over a wide range lattice constants for different coordination numbers in the laboratory. In fact, at criticality, because the expanded fluid has a much reduced coordination number, the lattice constant for expanded mercury is about $3 \AA$ across the metal to nonmetal transition. ${ }^{8}$ This agrees well with the experimental critical nearest-neighbor lattice constants of $2.9 \AA$ for $\mathrm{Hg}$ on $\mathrm{Ni}(111),{ }^{10,19} 3.16 \AA$ for $\mathrm{Hg}$ on $\mathrm{W}(100),{ }^{20}$ and $3.22 \AA$ for $\mathrm{Hg}$ on $\mathrm{Cu}(100){ }^{3}$

To explore the role of coordination number on the critical lattice constant for the nonmetal to metal transition, we undertaken model calculations of barium, a group II metal, in different geometries. Having a closed $n s^{2}$ shell configuration, group II metals are believed to be metals due to the band hybridization. Consequently, on increasing the lattice period, $a$, they should become insulators. The transition from metallic to nonmetallic state [usually considered in the reverse direction thus named the nonmetal-to-metal transition (NMT) $]$ corresponds to opening an absolute gap at $E_{F}$. Such a transition gives rise to a sharp drop (or rise for NMT) of density of states (DOS) at $E_{F}$, thus determining the lattice constant for criticality is readily accomplished. 

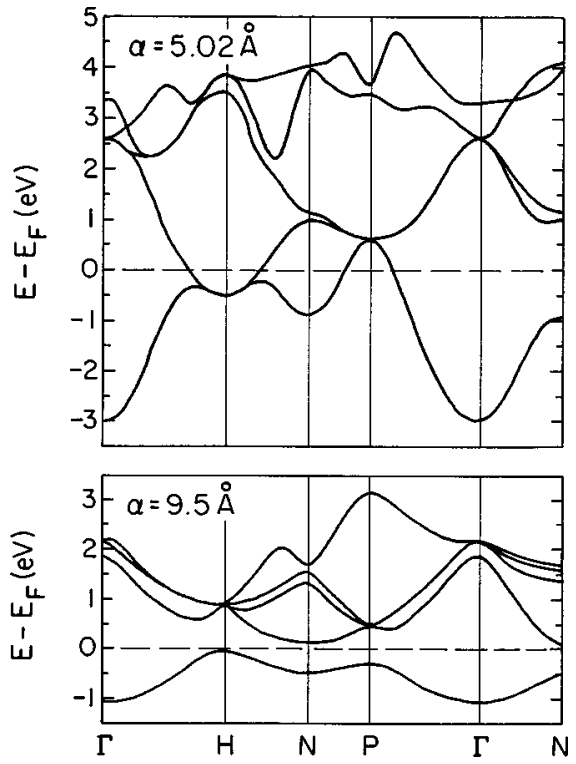

FIG. 1. Band structures for the bcc Ba crystal for lattice periods that correspond to the experimental value $(a=5.02 \AA)$ and the metallic state and to the nonmetallic state $(a=9.5 \AA)$. The nonmetal to metal transition occurs at $(a=7.9 \AA)$, see text.

\section{METHODOLOGY}

To elucidate the above issues concerning the role of the coordination number to the features of the nonmetal to metal transition in $2 \mathrm{D}$ and $3 \mathrm{D}$ systems, we have calculated the evolution of band structures for bulk and monolayer $\mathrm{Ba}$ on increasing lattice periods. Among the alkaline earth metals, Ba seems very attractive because of its rather close proximity to $\mathrm{Hg}$ whose metallization on decreasing the spacing is a classic example of the NMT. Thermodynamic arguments have been put forth that makes a compelling case that the metallicity in mercury depends strongly on coordination. ${ }^{4}$ Worth noting also, that for a metallic hexagonal closepacked $\mathrm{Ba}$ monolayer, the DOS is also extremely high ${ }^{21}$ which allows the studies of the sharpness of the transitions. The results on the evolution of the band structure for hexagonal $\mathrm{Ba}$ monolayers have been partly published elsewhere. ${ }^{22}$

The calculations have been performed by the scalar relativistic (film) linearized augmented-plane-wave (LAPW) method $^{23}$ assuming a bcc structure for the bulk Ba and hexagonal, square, and quasilinear structures for monolayers. In the latter case, a rectangular lattice with a longer period (the long length of the rectangle) of $a_{2}=10 \AA$ was employed to simulate the absence, or at least substantially diminished, electron density overlap between the linear chains, while the atomic separation along the chains $a_{1}$ was varied.

Using essentially the same methods for the bulk and the monolayers, the role of the dimensionality of the system can be directly studied by comparing the nearest-neighbor distances between $\mathrm{Ba}$ that correspond to the critical nonmetal to metal transition point.

\section{RESULTS}

The calculated bands for the bcc Ba for the lattice period $a=5.02 \AA$ (Fig. 1, top panel) are in a very good agreement
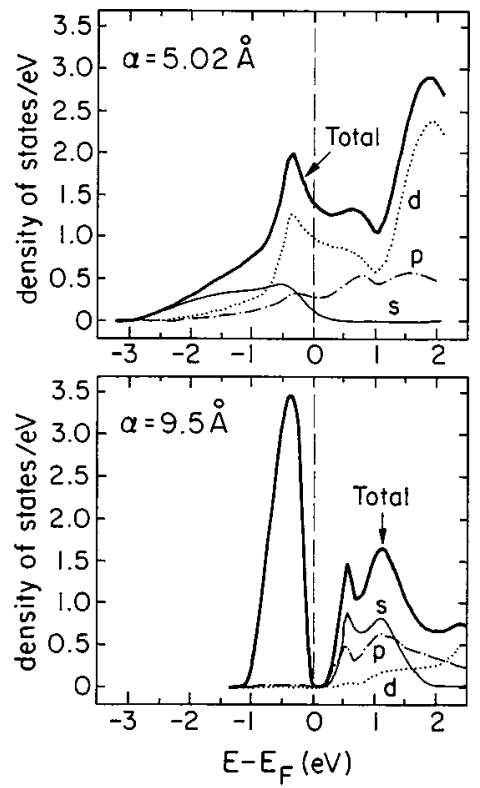

FIG. 2. Density of states for the bcc Ba for metallic and nonmetallic states in the metallic state $(a=5.02 \AA)$ and in the nonmetallic state $(a=9.5 \AA)$. The majority character of the bands is indicated as $s(-) ; p(-\cdot-) ; d(\cdots)$. Almost all of the occupied density of states in the insulating phase is $s$ in character (as expected).

with those for Eu (bcc, divalent, where the $f$ band is much lower) while the bandwidth below $E_{F}$ of $3.0 \mathrm{eV}$ is equal to reported for Ba by Chulkov and Silkin (using a relativistic pseudopotential). ${ }^{24}$ The essential role of the $s-p-d$ hybridization in the metallic state is evident from the top panel of Fig. 2. Namely, the density of states at the Fermi energy $D\left(E_{F}\right)$ is provided by the partial $s, p$, and $d$ yields. As the period increases, the relative position of the bands changes while they become narrower. This shift of the $p$ and $d$ bands (Fig. 2) relative to the $s$ band is most pronounced at the $N$ point (Fig. 1). On further increasing the lattice period, the shift of the $p$ and $d$ bands relative to the $s$ band increases until, at $a=9 \AA$, the gap opens thus indicating the transition to the nonmetallic state. This gap is evident in bottom panels of Figs. 1 and 2.

The transformations in electronic structure of Ba monolayers on increasing the period of a square lattice are similar to that for the bulk (Figs. 3 and 4). The $s-p-d$ hybridization gives rise to the metallic state for the lattice period $a=4.35$ $\AA$ which is twice the van der Waals radius for $\mathrm{Ba}$. The transition to the nonmetallic state, however, occurs at $a=5.7 \AA$ which is remarkably lower than the nearest-neighbor distance of $7.9 \AA$ for the transition in the bulk Ba.

Figure 5 illustrates evolution of the band structure for quasilinear Ba monolayers along $\overline{\Gamma X}$ which corresponds to the direction along the chains (there is little dispersion in $\overline{\Gamma Y}$ direction due to a large interchain separation). A quasi "onedimensional" metallicity for linear chains for $a=4.35 \AA$ changes to the dielectric state at the critical interatomic distance of $a=4.6 \AA$.

\section{DISCUSSION}

If both the lattice constant and coordination number both play a role in determining metallicity critically might be de- 


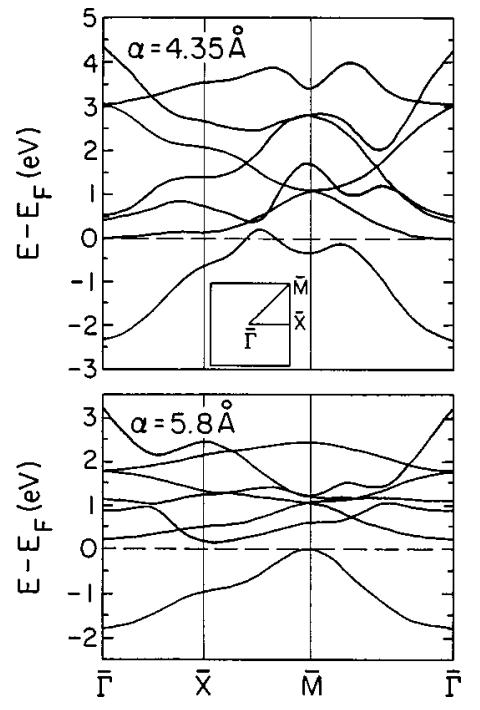

FIG. 3. Evolution of the band structure in the course of the metal to nonmetal transition in the Ba monolayers with a square lattice. The $a=4.35 \AA$ value corresponds to twice the van der Waals radius and a metallic state. The $a=5.8 \AA$ value corresponds to a nonmetallic state.

termined by a "critical" volume. Such a critical volume in an expanded fluid might be empirically related to that for thin films by

$$
(A / V) r_{0} \approx 1,
$$

where $A$ is the critical area per unit atom in an overlayer, $V$ is the critical volume in the expanded fluid, and $r_{0}$ is twice the van der Waals radius. A more accurate or sensible view might be to compare the Wigner-Seitz radius $\left(r_{s}\right.$ $\left.=3 V /(4 \pi)^{1 / 3}\right)$ to $1 / 2$ the critical nearest neighbor in the overlayer thin films. From the model calculations for barium, it is clear that such a simple scheme does not apply.

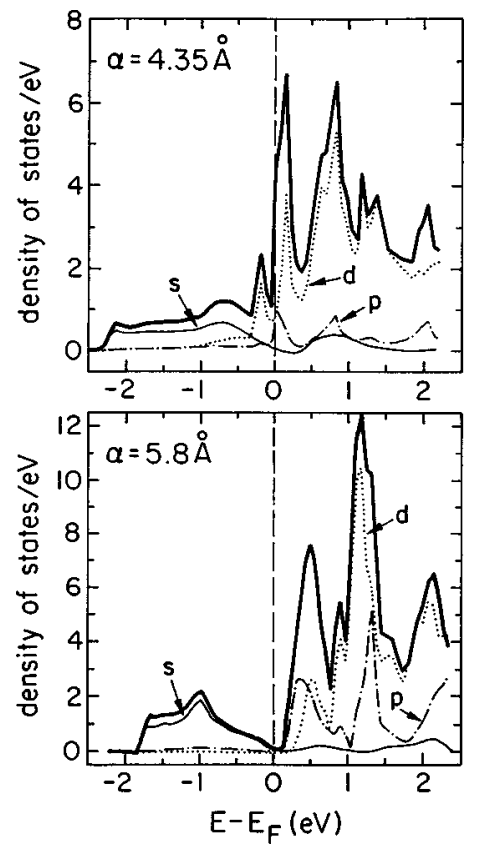

FIG. 4. DOS for $\mathrm{Ba}$ monolayers with a square lattice for metallic ( $a$ $=4.35 \AA)$ and nonmetallic $(a=5.8 \AA)$ states. The majority character of the bands is indicated as $s(-) ; p(-\cdot-) ; d(\cdots)$.
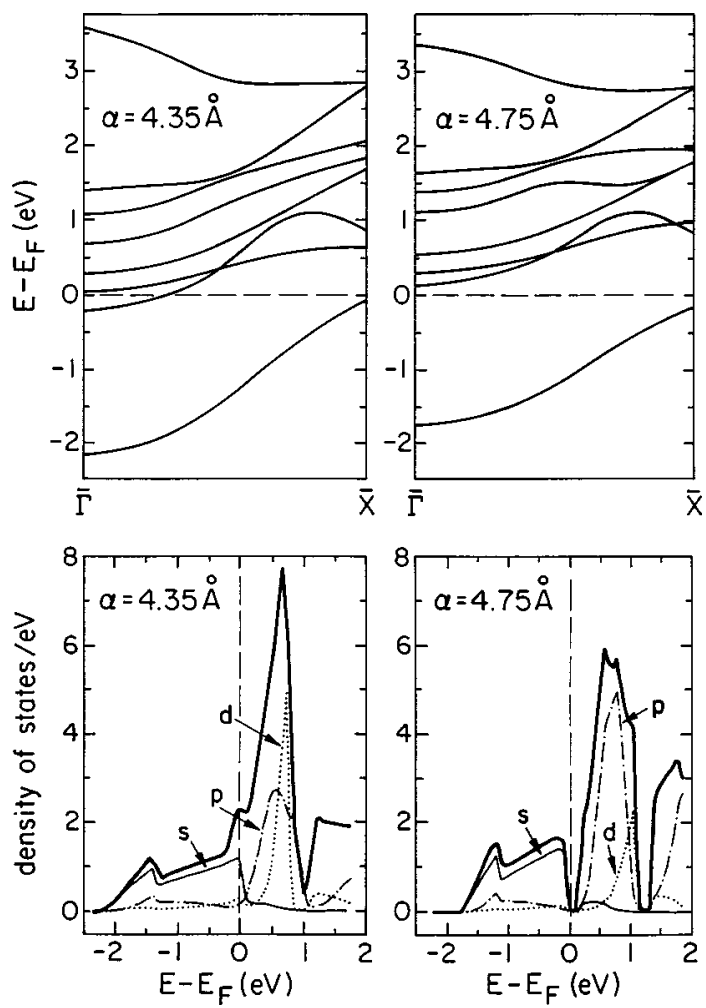

FIG. 5. The band structure (along the "chains") and density of states on either side of the metal to nonmetal transition for quasilinear Ba films. Both metallic $(a=4.35 \AA)$ and nonmetallic $(a=4.75 \AA)$ conditions are represented. The long length of the rectangle is $10 \AA$, see text. The majority character of the bands is indicated as $s(-) ; p(-\cdot-) ; d(\cdots)$ in the density of states.

The transition point for bec $\mathrm{Ba}, a=9 \AA$, corresponds to the nearest-neighbor distance of $7.9 \AA$ which much exceeds $5.7 \AA$ for the nonmetal to metal transition in the hexagonal $\mathrm{Ba}$ monolayers and square lattice Ba monolayers. The critical volume for bcc Ba is $365 \AA^{3}$, per atom. The critical volume per atom for the hexagonal monolayer of $\mathrm{Ba}$ is about a factor of 3 less $\left(121 \AA^{3}\right)$, as is the critical volume for the square lattice $\left(142 \AA^{3}\right)$. The quasi-one-dimensional lattice (the rectangular lattice) has a critical volume of $200 \AA^{3}$, so it is apparent there is no obvious or simple scaling behavior of the critical volume with coordination. Furthermore, it is also clear that there is no "universal" critical volume.

There is a generally larger critical nearest neighbor lattice constant associated with the larger coordination number in the bulk (7.9 $\AA$ ), a coordination of 8 for bcc Ba, as compared to 6 for the hexagonal monolayer (critical nearest neighbor spacing of $5.7 \AA$ ), 4 for the square lattice (critical nearest neighbor spacing of $5.7 \AA$ ), and approximately 2 for the rectangular lattice (critical nearest neighbor spacing of $4.6 \AA$ ). The comparison (Fig. 6) shows that the critical lattice constant does not scale as a simple inverse function of the coordination number. There does, however, seem to be a somewhat more transparent relationship between the critical nearest neighbor lattice constant and dimensionality.

Comparing the theory for the three-dimensional expanded mercury system(s) and the two-dimensional system(s) suggests that coordination also influences the critical lattice constants for the nonmetal to metal transition, but the 


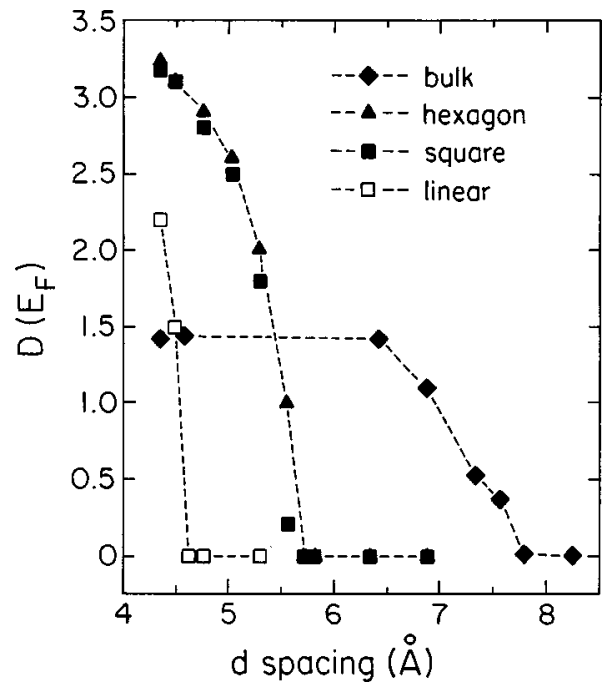

FIG. 6. Density of states at the Fermi level vs nearest-neighbor distance $d$ between Ba atoms for bulk bcc $(\bullet)$, hexagonal $(\boldsymbol{\Delta})$, square $(\boldsymbol{\square})$, and rectangular ( $\square$ ) (quasi-one-dimensional) lattices.

results are far less dramatic. The insulating phase can only be obtained if the lattice constant is 5.1-5.9 $\AA$ for fcc mercury and 4.2-5.0 $\AA$ for bcc mercury. ${ }^{25}$ The corresponding insulating state for the monolayer is about $3 \AA$ (Ref. 5) to $3.6 \AA{ }^{10}$ with little variation observed between the square packet lattice and hexagonal lattice in the latter calculation. ${ }^{10}$

It is not surprising that Singh and co-workers ${ }^{10}$ found that the influence of the lattice constant to be the dominant parameter in the nonmetal to metal transition and saw little influence of coordination in comparing only the square and hexagonal lattices. Our work with the Ba square and hexagonal lattices provides similar results to those for $\mathrm{Hg}$ in this regard but the importance of coordination, nonetheless, cannot be emphasized too much. Our work here shows that coordination (and perhaps, by extension, dimensionality) plays a dominant role in the nonmetal to metal transition, as is indeed suggested by the free cluster data. ${ }^{7}$ In the absence of structural characterization, it is impossible to do anything more than suggest that the increase in metallicity observed with increasing barium overlayer thickness on $\mathrm{Ni}(111)$ (Ref. 26) is a consequence of the increased average coordination number. Such an influence of coordination is certainly suggest by these calculations and would be similar to the change in metallicity observed for mercury overlayers. ${ }^{3}$

The expanded fluids differ from our (and other) calculation for the expanded 3D lattice in that the expanded fluids have low average coordination numbers, similar to the thin films, as we have noted. A tabulation of the experimental data suggests that since this latter comparison between three and two dimensions is for similar coordination numbers, the critical nearest neighbor lattice constant(s) are also similar. Thus a comparison of these different experimental systems is possible in a rough manner. Such an empirical comparison is attempted for molecular iodine, molecular bromine, mercury and the alkali metals as indicated in Table I. At first glance the values of $(A / V) r_{0}$ deviate considerably from unity, particularly in the case of cesium. On the other hand, surprisingly no value except that for cesium deviates from unity by
TABLE I. Experimental critical volumes and areas for the nonmetal to metal transitions.

\begin{tabular}{|c|c|c|c|c|}
\hline & $\begin{array}{c}\text { Critical volume } V \\
\left(\AA^{3} / \text { atom }\right)\end{array}$ & $\begin{array}{c}\text { Critical area } A \\
\left(\AA^{2} / \text { atom }\right)\end{array}$ & $\begin{array}{c}\text { van der Waals } \\
\text { diameter } \\
r_{0}(\AA)^{\mathrm{a}}\end{array}$ & $(A / V) r_{0}$ \\
\hline $\mathrm{Br}_{2}$ & $19.5^{\mathrm{b}}$ & $11.8^{\mathrm{d}}$ & 3.9 & 2.3 \\
\hline $\mathrm{I}_{2}$ & $26^{\mathrm{c}}$ & $14.5^{\mathrm{d}}$ & 4.3 & 2.4 \\
\hline $\mathrm{Hg}$ & 57 & $\begin{array}{r}8.38,{ }^{\mathrm{e}} 9.98,^{\mathrm{f}} \\
10.36^{\mathrm{g}}\end{array}$ & 3.005 & $0.44,0.52,0.54$ \\
\hline $\mathrm{Na}$ & 149 & $59^{32}$ & 3.82 & 1.51 \\
\hline $\mathrm{K}$ & 341 & $59^{32}$ & 4.70 & 0.81 \\
\hline CS & 579 & $25^{33}$ & 5.44 & 0.23 \\
\hline
\end{tabular}

${ }^{\mathrm{a}}$ Reference 34.

${ }^{\mathrm{b}}$ Reference 35 .

${ }^{\mathrm{c}}$ Reference 36.

${ }^{\mathrm{d}}$ Reference 28 .

${ }^{\mathrm{e}}$ Reference 19.

${ }^{\mathrm{f}}$ Reference 20 .

${ }^{\mathrm{g}}$ Reference 3.

more than $50 \%$. In passing it is important to note that it would be surprising if the values for $(A / V) r_{0}$ did not deviate from unity. The overlayers are investigated at temperatures well below the triple point in order to obtain chemisorption of the overlayer, furthermore, the substrates for the thin film overlayers are crystalline. This latter influence introduces order into the overlayer from the substrate potential corrugation, ${ }^{27}$ reducing the entropy beyond the influence of temperature alone. An interfacial entropy term correction is certainly indicated if the comparison is to be ever extended beyond the simply tantalizing.

Although bromine and iodine are among the most reactive systems listed in Table I, with the largest heats of adsorption for chemisorption, in fact they are among the least sensitive to the substrate corrugation. ${ }^{28}$ The halogen molecules are the most weakly adsorbed overlayers listed in Table I; they weakly molecularly chemisorb (associative adsorption). The poor agreement between molecular iodine and molecular bromine and the nonmetal to metal transition may be a consequence of the fact that for these two systems the overlayers have never been compressed enough to drive them through a nonmetal to metal transition. Greater compression of the overlayer would act to reduce the critical $(A / V) r_{0}$ ratio.

\section{SUMMARY}

Theory makes a compelling case that there is no universal "critical volume" per atom for the nonmetal to metal transition. Rather, we find that coordination is a dominant factor (along with nearest neighbor spacing, of course) in determining the nonmetal to metal transition, as has been suggested before. ${ }^{1-7}$ If the comparison between expanded fluids and thin film overlayers is indeed possible then the expanded fluids cannot be close packed 3D lattice(s) and that the reduction in coordination is key to the metal to nonmetal transition. The comparison of three-dimensional systems and two-dimensional systems may only be possible when the coordination number is similar. There are clearly a number of issues that should be investigated if we are to accomplish the 
goal of comparing the nonmetal to metal transition empirically in two and three dimensions. Still unresolved and not addressed here are the questions about the role of the substrate on the nonmetal to metal transition in ultrathin film overlayers.

What is indicated is the need for both more experiments and more theories. The influence of the substrate on the nonmetal to metal transition in overlayers must be rationalized. Experimental studies of expanded fluid lithium, $\mathrm{Mg}, \mathrm{Sr}$, and $\mathrm{Ba}$ should be undertaken to compare with the nonmetal to metal transition of lithium on $\mathrm{Be}(0001),{ }^{29} \mathrm{Mg}$ on $\mathrm{Mo}(112),{ }^{30,31} \mathrm{Sr}$ on $\mathrm{Mo}(112),{ }^{31}$ and $\mathrm{Ba}$ on $\mathrm{Mo}(112) .^{31-33}$ More structural studies are indicated for the expanded fluids and are necessary for comparison of critical coordination numbers. If there is to be an extension of this comparison of the nonmetal to metal transition to clusters, then studies of free clusters of $\mathrm{Mg}, \mathrm{Br}_{2}, \mathrm{I}_{2}, \mathrm{Sr}$, and $\mathrm{Ba}$ are indicated, while surface studies and expanded fluid studies of $\mathrm{Zn}$ and $\mathrm{Cd}$ through the nonmetal to metal transition may prove valuable.

\section{ACKNOWLEDGMENTS}

The authors would like to thank a number of their colleagues, in particular F. Hensel, Sergei Baranouski, and R. Winter. The subject originally arose in discussions at Phillips University of Marburg with F. Hensel. The research program of one of us (P.A.D.) is supported by the National Science Foundation through Grant No. DMR-9802126.

${ }^{1}$ G. R. Freeman and N. H. March, J. Phys. Chem. 98, 9486 (1994).

${ }^{2}$ N. H. March, M. P. Tosi, and D. J. Klein, Phys. Rev. B 52, 9115 (1995).

${ }^{3}$ P. A. Dowben, D. LaGraffe, D. Li, G. Vidali, L. Zhang, L. Dottl, and M. Onellion, Phys. Rev. B 43, 10677 (1991).

${ }^{4}$ A. R. Miedema and J. W. F. Dorleijn, Philos. Mag. B 43, 251 (1981).

${ }^{5}$ H. J. F. Jansen, A. J. Freeman, M. Weinert, and E. Wimmer, Phys. Rev. B 28, 593 (1983)

${ }^{6}$ P. A. Dowben, Surf. Sci. Rep. (2000), in press.

${ }^{7}$ P. A. Dowben, D. N. McIlroy, Jiandi Zhang, and E. Rühl, Mater. Sci. Eng., A 217/218, 258 (1996).

${ }^{8}$ K. Tamura and S. Hosokawa, J. Phys.: Condens. Matter 6, A241 (1991); K. Tamura, M. Inui, I. Nakaso, Y. Oh'Ishi, K. Funakoshi, and W. Utsumi, ibid. 10, 11405 (1998).

${ }^{9}$ R. Winter, F. Noll, T. Bodensteiner, W. Gläser, P. Chieux, and F. Hensel, Z. Phys. Chem. (Munich) 156, 145 (1988).

${ }^{10}$ N. K. Singh, P. Dale, D. Bullett, and R. G. Jones, Surf. Sci. 294, 333 (1994).
${ }^{11}$ Jiandi Zhang, Dongqi Li, and P. A. Dowben, Phys. Lett. A 173, 183 (1993).

${ }^{12}$ Jiandi Zhang, Dongqi Li, and P. A. Dowben, J. Phys.: Condens. Matter 6, 33 (1994).

${ }^{13}$ K. Rademann, B. Kaiser, U. Even, and F. Hensel, Phys. Rev. Lett. 59, 2319 (1987)

${ }^{14}$ C. Brechignac, M. Broyer, Ph. Cahuzac, G. Delcretaz, P. Labastie, J. P. Wolf, and L. Wöste, Phys. Rev. Lett. 60, 275 (1988).

${ }^{15}$ H. Haberland, H. Kornmeier, H. Langosch, M. Oschwald, and G. Tanner, J. Chem. Soc., Faraday Trans. 86, 2473 (1990).

${ }^{16}$ R. E. Benfield, J. Chem. Soc., Faraday Trans. 88, 1107 (1992).

${ }^{17}$ M. Onellion, Y. J. Kime, P. A. Dowben, and N. Tache, J. Phys. C 20, L633 (1987).

${ }^{18}$ P. A. Dowben, Shikha Varma, Y. J. Kime, D. R. Mueller, and M. Onellion, Z. Phys. B 73, 247 (1988).

${ }^{19}$ N. K. Singh and R. G. Jones, Chem. Phys. Lett. 155, 463 (1989); Surf. Sci. 232, 243 (1990).

${ }^{20}$ W. F. Egelhoff, Jr., D. L. Perry, and J. W. Linnett, Surf. Sci. 54, 670 (1976)

${ }^{21}$ E. Wimmer, J. Phys. F 14, 681 (1984).

${ }^{22}$ I. N. Yakovkin, Surf. Sci. 442, 431 (1999).

${ }^{23}$ H. Krakauer, M. Posternak, and A.-J. Freeman, Phys. Rev. B 19, 1706 (1979); M. Posternak, H. Krarauer, A.-J. Freeman, and D. D. Koelling, ibid. 21, 5601 (1980).

${ }^{24}$ E. V. Chulkov, V. M. Silkin, and I. Yu. Sklyadneva, Surf. Sci. 231, 9 (1990).

${ }^{25}$ F. Yonezawa and T. Ogawa, Suppl. Prog. Theor. Phys. 72, 1 (1982).

${ }^{26}$ P. A. Dowben and D. LaGraffe, Phys. Lett. A 144, 193 (1990).

${ }^{27}$ W. Li, J.-S. Lin, M. Karimi, P. A. Dowben, and G. Vidali, Phys. Rev. B 45, 3708 (1992)

${ }^{28}$ This is the critical area per molecule (not per atom) based upon the closed packed lattice of iodine and bromine from D. Mueller, T. N. Rhodin, and P. A. Dowben, Ber. Bunsenges. Phys. Chem. 90, 281 (1986), assuming an upright molecular bonding configuration as noted in D. Mueller, T. N. Rhodin, and P. A. Dowben, Surf. Sci. 164, 271 (1985); P. A. Dowben, Crit. Rev. Solid State Mater. Sci. 13, 191 (1987)

${ }^{29}$ G. M. Watson, P. A. Bruhwiler, H. J. Sanger, K. H. Frank, and E. W. Plummer, Phys. Rev. B 50, 17678 (1994).

${ }^{30}$ J. Zhang, D. N. McIlroy, and P. A. Dowben, Phys. Rev. B 49, 13780 (1994); 52, 11380 (1995); Europhys. Lett. 29, 469 (1995).

${ }^{31}$ G. A. Katrich, V. V. Klimov, and I. N. Yakovkin, Ukr. Phys. J. 36, 722 (1991); J. Electron Spectrosc. Relat. Phenom. 68, 369 (1994).

${ }^{32}$ D. M. Riffe, G. K. Wertheim, J. E. Rowe, and P. H. Citrin, Phys. Rev. B 45, 3532 (1992)

${ }^{33}$ N. J. DiNardo, T. M. Wong, and E. W. Plummer, Phys. Rev. Lett. 65, 2177 (1990).

${ }^{34}$ A. Bondi, J. Phys. Chem. 68, 441 (1964); or deduced from equilibrium lattice constants tabulated in CRC Handbook of Chemistry and Physics, 75th ed., edited by D. R. Lide (Chemical Rubber, Cleveland, 1995).

${ }^{35}$ H. Fujihisa, Y. Fuji, K. Takemura, and O. Shimomura, J. Phys. Chem. Solids 56, 1439 (1995).

${ }^{36}$ K. Takemura et al., Phys. Rev. B 26, 998 (1982). 